\title{
Uso do teorema de ponto fixo de Banach para a determinação de solução de uma Equação do tipo de Kirchhoff
}

\author{
Douglas B. Costa* André L. M. Martinez Marcelo R. A. Ferreira \\ Coordenação de Matemática, COMAT, UTFPR, \\ 86300-000, Cornélio Procópio, PR \\ E-mail: britocostadouglas@gmail.com, andrelmmartinez@yahoo.com, marcelorafereira@gmail.com.
}

\section{$\underline{\text { RESUMO }}$}

Neste trabalho faremos uso do Teorema de Banach para demonstrar a existência e a unicidade de solução para uma equação do tipo de Kirchhoff. A equação apresentada a seguir possui aplicações no estudo de vibrações livres em cordas elásticas (recomendamos[1], [3] e [4]):

$$
\left\{\begin{array}{l}
-M\left(\left\|u^{\prime}\right\|_{2}^{2}\right) u^{\prime \prime}=f\left(t, u, u^{\prime}\right) \\
u(0)=u(1)=0
\end{array}\right.
$$

onde $M: \mathbb{R}^{+} \rightarrow \mathbb{R}^{+}$e $f:[0,1] \times \mathbb{R} \times \mathbb{R}$. Vamos determinar solução para (1) baseado nos resultados apresentados em [2], trabalharemos no espaço de Banach $E=\left\{u \in C^{\prime}[0,1] ; u(0)=u(1)=0\right\}$ com a norma $\|u\|_{E}=\left\|u^{\prime}\right\|_{\infty}=\max _{t \in[0,1]}\left|u^{\prime}(t)\right|$. As soluções de (1) podem ser escritas da seguinte forma: $u(t)=\int_{0}^{1} G(t, s) \frac{f\left(s, u(s), u^{\prime}(s)\right)}{M\left(\left\|u^{\prime}\right\|_{2}^{2}\right)} d s$, onde $G(t, s)=\left\{\begin{array}{l}t-t s, t \leq s ; \\ s-s t, s \leq t .\end{array}\right.$ Deste modo $u$ é solução de (1) se for ponto fixo do operador $T: E \rightarrow E$ definido por:

$$
(T u)(t)=\int_{0}^{1} G(t, s) \frac{f\left(s, u(s), u^{\prime}(s)\right)}{M\left(\left\|u^{\prime}\right\|_{2}^{2}\right)} d s .
$$

Vamos estabelecer a existência e unicidade de solução, para isto consideremos a sequência iterativa

(i) $u^{k+1}=T\left(u^{k}\right)$

(ii) $u^{k+1}(t)=\int_{0}^{1} G(t, s) \frac{f\left(s, u_{k}(s), u_{k}^{\prime}(s)\right)}{M\left(\left\|u_{k}^{\prime}\right\|_{2}^{2}\right)} d s$.

As seguintes hipóteses serão necessárias:

(S1) Existem constantes positivas $\alpha, A$ e $B$ tais que $A \leq M\left(\left\|u^{\prime}\right\|_{2}^{2}\right) \leq B, \forall u \in E$, com $\|u\|_{E} \leq \alpha$.

(S2) $\alpha$ e A cumprem $\max _{(s, u, v) \in[0,1] \times\left[-\frac{\alpha}{2}, \frac{\alpha}{2}\right] \times[-\alpha, \alpha]}|f(s, u, v)| \leq \frac{\alpha A}{d_{1}}$; onde $d_{1}=\max _{t}\left\{\int_{0}^{1}\left|\frac{\partial G(t, s)}{\partial t}\right| d s\right\}$.

(S3) Existe $\lambda_{M}>0$, tal que $|M(u)-M(v)| \leq \lambda_{M}|u-v|, \forall u, v \in[-\alpha, \alpha]$;

(S4) Existe $\lambda_{f}>0 \mathrm{com}\left|f\left(s, u, u^{\prime}\right)-f\left(s, v, v^{\prime}\right)\right| \leq \lambda_{f} \max |u-v|,\left|u^{\prime}-v^{\prime}\right|$ onde $s \in[0,1], u, v \in$ $\left[-\frac{\alpha}{2}, \frac{\alpha}{2}\right]$ e $u^{\prime}, v^{\prime} \in[-\alpha, \alpha]$

(S5) Vale a seguinte desigualdade $\frac{2 \lambda_{M} \alpha^{2}}{A}+\frac{B \lambda_{f} d_{1}}{A^{2}}<1$.

Teorema 1. Suponha que (S1)-(S5) ocorra. Então (1) possui solução iterativa com $\|u\|_{E} \leq \alpha$.

\footnotetext{
${ }^{*}$ Bolsista de Iniciação Científica PIBIC-UTFPR
} 
Demonstração: Primeiramente, mostraremos que $T: B[0, \alpha] \rightarrow B[0, \alpha]$, onde $B[0, \alpha]=\left\{u \in E ;\|u\|_{E} \leq \alpha\right\}$. De fato, se $u \in B[0, \alpha]$, temos:

$\left\|(T u)^{\prime}\right\|_{\infty}=\max _{t}\left\{\left|\int_{0}^{1} \frac{\partial G(t, s)}{\partial t} \frac{f\left(s, u(s), u^{\prime}(s)\right)}{M\left(\left\|u^{\prime}\right\|_{2}^{2}\right)} d s\right|\right\} \leq \max _{s}\left|f\left(s, u, u^{\prime}\right)\right| \frac{1}{M\left(\left\|u^{\prime}\right\|_{2}^{2}\right)} \int_{0}^{1}\left|\frac{\partial G(t, s)}{\partial t} d s\right|$,

de (S1) e (S2) obtemos: $\left\|(T u)^{\prime}\right\|_{\infty} \leq\left(\frac{\alpha A}{d_{1}}\right) \frac{1}{M\left(\left\|u^{\prime}\right\|_{2}^{2}\right)} d_{1} \leq \frac{\alpha A}{d_{1}}\left(\frac{1}{A}\right) d_{1}=\alpha \Rightarrow\left\|(T u)^{\prime}\right\|_{\infty} \leq \alpha$. Portanto, $T: B[0, \alpha] \rightarrow B[0, \alpha]$. Vamos mostrar que $T$ é uma contração e então obter o resultado do Teorema do Ponto Fixo de Banach. Segue de (S2), (S3) e (S4) que

$$
\begin{aligned}
\left\|(T u-T v)^{\prime}\right\|_{\infty} & =\max _{t}\left\{\left|\int_{0}^{1} \frac{\partial G(t, s)}{\partial t}\left[\frac{f\left(s, u(s), u^{\prime}(s)\right)}{M\left(\left\|u^{\prime} \mid\right\|_{2}^{2}\right)}-\frac{f\left(s, v(s), v^{\prime}(s)\right)}{M\left(\| v^{\prime}||_{2}^{2}\right)}\right] d s\right|\right\} \\
& \leq d_{1} \max _{s}\left\{\left|\frac{f\left(s, u(s), u^{\prime}(s)\right.}{M\left(\left\|u^{\prime}\right\|_{2}^{2}\right)}-\frac{f\left(s, v(s), v^{\prime}(s)\right)}{M\left(\left\|v^{\prime}\right\|_{2}^{2}\right)}\right|\right\} \\
& \leq d_{1} \frac{1}{A^{2}} \max _{s} \mid M\left(\left\|v^{\prime}\right\|_{2}^{2}\right) f\left(s, u(s), u^{\prime}(s)\right)-M\left(\left\|u^{\prime}\right\|_{2}^{2}\right) f\left(s, v(s), v^{\prime}(s)\right) \\
& \leq \frac{d_{1}}{A^{2}}\left[\left|M\left(\left\|v^{\prime}\right\|_{2}^{2}\right)-M\left(\left\|u^{\prime}\right\|_{2}^{2}\right)\right| \max _{s}\left|f\left(s, u(s), u^{\prime}(s)\right)\right|+\right. \\
& \leq \frac{d_{1}}{A^{2}}\left[\left.\lambda_{M}\|\|^{\prime}\left\|_{2}^{2}-\right\|\right|_{2} ^{2}\right) \max _{s} \mid f\left(s, u(s), u_{2}^{2}\left(\frac{\alpha A}{d_{1}}\right)+B \lambda_{f}\left\|u^{\prime}-v^{\prime}\right\|_{\infty}\right] .
\end{aligned}
$$

Note que ||$\left|v^{\prime}\left\|_{2}^{2}-\right\| u^{\prime} \|_{2}^{2}\right|=\left|\int_{0}^{1}\left(\left[v^{\prime}(s)\right]^{2}-\left[u^{\prime}(s)\right]^{2}\right) d s\right| \leq \int_{0}^{1}\left|\left[v^{\prime}(s)+u^{\prime}(s)\right]\left[v^{\prime}(s)-u^{\prime}(s)\right]\right| d s \leq$ $\left\|u^{\prime}+v^{\prime}\right\|_{\infty}\left\|u^{\prime}-v^{\prime}\right\|_{\infty}$, assim

$$
\begin{aligned}
\left\|(T u-T v)^{\prime}\right\|_{\infty} & \leq \frac{d_{1}}{A^{2}}\left[\lambda_{M}\left\|u^{\prime}+v^{\prime}\right\|_{\infty}\left\|u^{\prime}-v^{\prime}\right\|_{\infty}\left(\frac{\alpha A}{d_{1}}\right)+B \lambda_{f}\left\|u^{\prime}-v^{\prime}\right\|_{\infty}\right] \\
& \leq \frac{d_{1}}{A^{2}}\left[\lambda_{M}(2 \alpha)\left(\left\|u^{\prime}-v^{\prime}\right\|_{\infty}\right)\left(\frac{\alpha A}{d_{1}}\right)+B \lambda_{f}\left\|u^{\prime}-v^{\prime}\right\|_{\infty}\right] \\
& \leq\left(\frac{2 \lambda_{M} \alpha^{2}}{A}+\frac{B \lambda_{f} d_{1}}{A^{2}}\right)\left\|u^{\prime}-v^{\prime}\right\|_{\infty} .
\end{aligned}
$$

Segue de (S5) que $T: B[0, \alpha] \rightarrow B:[0, \alpha]$ é uma contração.

Este estudo encontra-se em fase inicial e pretendemos além de explorar os resultados teóricos fornecidos pelo Teorema de Ponto Fixo de Banach vamos também realizar testes numéricos com algoritmos baseados na sequencia iterativa definida em (2).

Palavras-chave: Equação de Kirchhoff, Ponto fixo, Teorema de Banach

\section{Referências}

[1] R.P. Agarwal, M. Meehan e D.O'Regan. "Fixed Point Theory and Applications". Cambridge University Press, Cambridge (2011).

[2] A.L.M. Martinez, E.V.Castelani, J.da Silva, W.V.IShirabayashi A note about positive solutions for an equation of Kirchhoff type, Applied Mathematics and Computation, 218 (2011) 2082-2090.

[3] R.K. Sharma, Iteractive solutions to boundary-value differential equations with reflection of the argument, J. Comp. Appl. Math. 24(1988) 319-326.

[4] T.F. Ma, E.S. Miranda, A nonlinear differencial equation involving reflection of the argument, Arch. Math. 40 (2004) 63-68.

[5] T.F. Ma, Remarks on an elliptic equation of Kirchhoff type, Nonlinear Analysis (63), 2005, 19671977. 